\title{
A new convenient access to highly functionalized (E)-2-arylvinyl bromides
}

\author{
YUBO JIANG and CHUNXIANG KUANG* \\ Department of Chemistry, Tongji University, Siping Road 1239, Shanghai 200092, China \\ e-mail: kuangcx@tongji.edu.cn
}

MS received 13 November 2008; 11 February 2009; accepted 16 April 2009

\begin{abstract}
Highly functionalized (E)-2-arylvinyl bromides were prepared in high yields through a new convenient access by acylation of $(E)$-4-(2-bromovinyl)phenol with fatty and aromatic acids at room temperature using dicyclohexyl carbodiimide (DCC) and dimethylaminopyridine (DMAP).
\end{abstract}

Keywords. (E)-2-Arylvinyl bromide; acylation; deacetylation.

\section{Introduction}

(E)-2-Arylvinyl bromides are important building blocks in organic synthesis, especially as intermediates for carbon-carbon and carbon-hetero atom bond formation by transition metal catalysed coupling reactions. ${ }^{1,2}$ The coupling products from functionalized $(E)$-2-arylvinyl bromides have found numerous applications in the preparation of pharmaceuticals, functional polymeric material and natural products. Synthetic routes to these compounds are limited and mainly involve Hunsdiecker-type bromodecarboxylation of substituted trans-cinnamic acids with bromine sources such as $\mathrm{NBS}^{3-6} \mathrm{Br}^{+}(\text {coll })_{2} \mathrm{PF}_{6}^{-7}{ }^{7} \mathrm{Br}^{-} /$ oxidant. ${ }^{8-13}$ However, these synthetic methods could not afford good yields and high $E / Z$ stereoselectivities when the substrate bearing functional groups.

In this paper, we report a new convenient access to highly functionalized (E)-2-arylvinyl bromides (6) bearing various functional groups in high yields by acylation of $(E)$-4-(2-bromovinyl)phenol (4) with fatty acid and aromatic acid (5) at room temperature using dicyclohexyl carbodiimide (DCC) and dimethylaminopyridine (DMAP) (scheme 1). To the best of our knowledge, efficient synthetic method of (E)-2-arylvinyl bromides bearing various groups has not been reported.

*For correspondence

\section{Experimental}

\subsection{Materials and reagents}

Melting points were recorded using a A. Krüss Optronic GmbH KSPII melting-point meters and were uncorrected. IR spectra were performed on a Nexus FT-IR spectrophotometer. ${ }^{1} \mathrm{H}$ and ${ }^{13} \mathrm{C}$ NMR spectra were recorded using a Bruker AM-300 spectrometer. Elemental analyses were performed with a Perkin-Elmer 2400 CHNS elemental analyzer. Mass spectra were recorded by EI, MALDI, and ESI methods. All reactions were monitored by TLC with Huanghai GF254 silica gel coated plates. Flash column chromatography was carried out using 300-400 mesh silicagel at medium pressure. $p$-Acetoxycinnamic acid 2 was prepared according to literature procedures. $^{14}(E)$-4-(2-bromovinyl)phenyl acetate $\mathbf{3}$ was also prepared according to literature procedures $^{6}$ and 3 was directly converted to $(E)-4-$ (2-bromovinyl)phenol 4 by selective cleavage of the acetyl protecting group in the presence of EtONa. ${ }^{6}$ Other materials were obtained from commercial sources.

\subsection{General procedure for the synthesis of 6}

(E)-4-(2-bromovinyl)phenol $(4,1.0 \mathrm{mmol}), \mathrm{RCO}_{2} \mathrm{H}$ $(5,1.1 \mathrm{mmol})$ and DMAP $(1.1 \mathrm{mmol})$ in anhydrous benzene $(15 \mathrm{~mL})$ were stirred for $10 \mathrm{~min}$. To the reaction mixture was added DCC $(1.1 \mathrm{mmol})$ and stirred for 2-24 h at ambient temperature. After the completion of the reaction (monitored by TLC, 


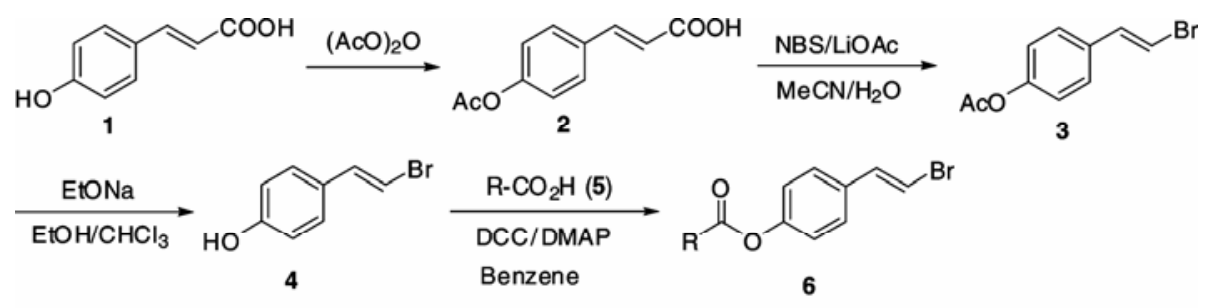

$\mathrm{R}=$ alkyl or aryl groups bearing $-\mathrm{CHO},-\mathrm{CO}_{2} \mathrm{R}$, styryl, ether, vinyl bromide, etc.

Scheme 1. Synthesis of functionalized (E)-2-arylvinyl bromides 6 .

Table 1. Synthesis of functionalized (E)-2-arylvinyl bromides.

\begin{tabular}{|c|c|c|c|c|c|c|c|}
\hline Entry & & $\mathrm{O}_{2} \mathrm{H}$ & & Product 6 & & Time (h) & Yield of $6(\%)^{a, b}$ \\
\hline 1 & $n-\mathrm{C}_{15} \mathrm{H}_{31}-\mathrm{CO}_{2} \mathrm{H}$ & $5 a$ & & & $6 a$ & 2 & 99 \\
\hline 2 & $\leadsto \mathrm{CO}_{2} \mathrm{H}$ & $5 b$ & & & $6 b$ & 2 & 99 \\
\hline 3 & & $5 c$ & & & $6 c$ & 24 & 87 \\
\hline 4 & & $\begin{array}{l}5 d \\
5 e\end{array}$ & $\begin{array}{l}\mathrm{R}^{1} \\
-\mathrm{H} \\
-\mathrm{CO}_{2} \mathrm{Me}\end{array}$ & & $\begin{array}{l}\text { 6d } \\
6 e\end{array}$ & $\begin{array}{l}24 \\
24\end{array}$ & $\begin{array}{l}98 \\
88\end{array}$ \\
\hline 5 & & $\begin{array}{l}5 f \\
5 g \\
5 h\end{array}$ & 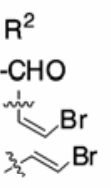 & & $\begin{array}{l}6 f \\
6 \mathrm{~g} \\
6 \mathrm{~h}\end{array}$ & $\begin{array}{r}24 \\
3 \\
4\end{array}$ & $\begin{array}{l}86 \\
93 \\
95\end{array}$ \\
\hline 6 & & $5 \mathbf{i}$ & & & $6 i$ & 24 & 97 \\
\hline 7 & & $5 \mathbf{j}$ & & & 6j & 24 & 87 \\
\hline 8 & & $\begin{array}{l}5 k \\
51\end{array}$ & $\begin{array}{l}X \\
\text { o } \\
\text { s }\end{array}$ & & $\begin{array}{l}6 k \\
61\end{array}$ & $\begin{array}{r}5 \\
24\end{array}$ & $\begin{array}{l}98 \\
94\end{array}$ \\
\hline
\end{tabular}

${ }^{\mathrm{a}}$ Isolated yields; ${ }^{\mathrm{b}} E / Z:>99 / 1$, determined by ${ }^{1} \mathrm{H}$ NMR analysis

EtOAc: hexane, 1:2), the solvent was evaporated and the residue was subjected to column chromatography (silica gel, EtOAc-petroleum ether, 1:8$1: 4)$ to afford functionalized 4-acyloxy-(E)-2arylvinyl bromides $\mathbf{6 a}-\mathbf{l}$ (table 1).

\section{Results and discussion}

\subsection{Synthesis of (E)-4-(2-bromovinyl)phenol 4}

For our initial studies, $p$-hydroxy cinnamic acid (1) was chosen as starting material in the presence of
NBS to generate (E)-4-(2-bromovinyl)phenol (4) directly, but unexpected by-product dibromide was always observed owing to the electron-donating group of hydroxyl group, and it was very difficult to separate. Thus, we attempted to acetylation of hydroxyl group before bromination in the presence of NBS and subsequent deacetylation, and $(E)-4-(2-$ bromovinyl)phenol (4) was obtained in high yield with high stereoselectivity (scheme 1).

Interestingly, a mixture of (E)-4-(2-bromovinyl) phenyl acetate 3 and $\alpha$-bromo- $\beta$-lactone $3^{\prime}$ were found in this reaction of 2 with NBS (scheme 2). 


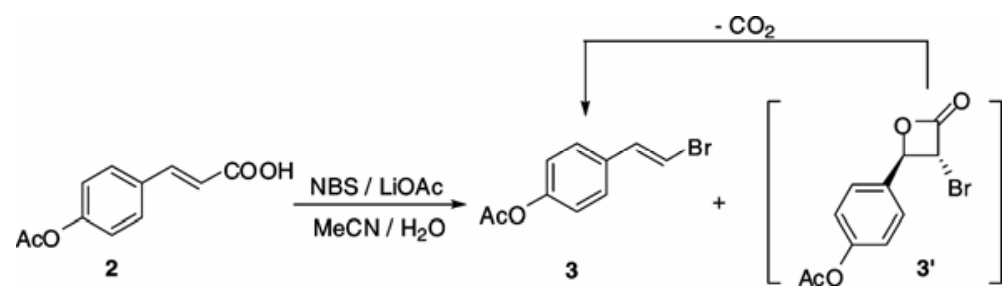

Scheme 2. Formation of (E)-4-(2-bromovinyl) phenyl acetate.

Table 2. Esterification condition of acetic acid with (E)-4-(2-bromovinyl)phenol (4).

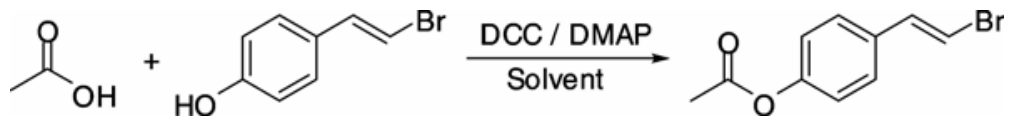

\begin{tabular}{ccclc} 
Entries & $\mathrm{CH}_{3} \mathrm{CO}_{2} \mathrm{H}$ equiv. & DCC/DMAP equiv. & Solvent & Yield (\%) ${ }^{\mathrm{a}, \mathrm{b}}$ \\
\hline 1 & 1 & $1 /$ cat. & $\mathrm{DMF}$ & $<5$ \\
2 & 1 & $1 / 0 \cdot 5$ & $\mathrm{DMF}$ & 16 \\
3 & 1 & $1 / 1$ & $\mathrm{DMF}$ & 32 \\
4 & $1 \cdot 1$ & $1 \cdot 1 / 1 \cdot 1$ & $\mathrm{DMF}$ & 41 \\
5 & $1 \cdot 1$ & $1 / 1$ & $\mathrm{CH}_{2} \mathrm{Cl}_{2}$ & 35 \\
6 & $1 \cdot 1$ & $1 \cdot 1 / 1 \cdot 1$ & $\mathrm{CH}_{3} \mathrm{Cl}_{2}$ & 47 \\
7 & 1 & $1 \cdot 1 / 1$ & Benzene & 85 \\
8 & $1 \cdot 1$ & $1 \cdot 1 / 1 \cdot 1$ & Benzene & 99 \\
9 & $1 \cdot 1$ & $1 \cdot 1 /$ cat. & Benzene & 23 \\
10 & $1 \cdot 1$ & $1 \cdot 1 / 1 \cdot 1$ & Toluene & 49 \\
\hline
\end{tabular}

${ }^{a}$ Isolated yields. ${ }^{b} E / Z:>99 / 1$, determined by ${ }^{1} \mathrm{H}$ NMR analysis

The coupling constant $(J=3.96 \mathrm{~Hz})$ observed in the ${ }^{1} \mathrm{H}$ NMR of $\mathbf{3}^{\prime}$ indicated that $\mathbf{3}^{\prime}$ was a trans- $\beta$ lactone. ${ }^{6}$ In the IR spectrum, the characteristic carbonyl absorption was observed at $1810 \mathrm{~cm}^{-1}(\beta$ lactone $\mathrm{C}=\mathbf{O}$ ). In HRMS, the $m / z$ value was found as 283.9684, compared with the calculated 283.9683. Separating $\beta$-lactone from this mixture by column chromatography was difficult since $\mathbf{3}^{\prime}$ was unstable to elimination carbon dioxide to $\mathbf{3}$ even in room temperature as shown by ${ }^{1} \mathrm{H}$ NMR detection.

\subsection{Synthesis of functionalized (E)-2-arylvinyl bromides 6}

The esterification of phenols using anhydrides or acyl halides as esterifying agent is one of the most routinely used transformation in organic synthesis. While this method was applied in the synthesis of 4-acyloxy-(E)-2-arylvinyl bromides, the yield was poor mainly due to the formation of by-product under condition of acid or high temperature. In order to avoid the formation of by-product, a mild reaction condition should be applied. We tried different activating reagents for the esterification of $(E)-4-(2-$ bromovinyl)phenol (4) and found only DCC/DMAP offered high yields, while others such as DCC alone, DCC/HOBT, and BOP resulted in low yields $(<15 \%)$.

To optimize this esterification conditions, the reaction of (E)-4-(2-bromovinyl)phenol (4) with acetic acid was carried out. We tested several solvents including DMF, $\mathrm{CH}_{2} \mathrm{Cl}_{2}$, and toluene, but they afforded very low yields. As further work was carried out, we found satisfying yield when benzene and $1 \cdot 1$ equivalent DMAP revolved. The $E / Z$ ratio of the products did not vary under this reaction conditions, and the geometry of the $\mathrm{C}-\mathrm{C}$ double bond was retained during the reaction procedure (table 2 ).

The best condition for the esterification of $(E)-4$ (2-bromovinyl)phenol (4) was $1 \cdot 1$ equivalent DCC and 1.1 equivalent DMAP in benzene as solvent. When DMAP was used in catalytic amount the yield was drastically decreased $(<25 \%$, table 2 , entries 1 , 9). It is crucial that DCC should be added after all the other reagents had been added and stirred for ten munites. The rusults are shown in table 2 .

These results indicated that the present reaction was very useful for the synthesis of both 4-alkoxy- 


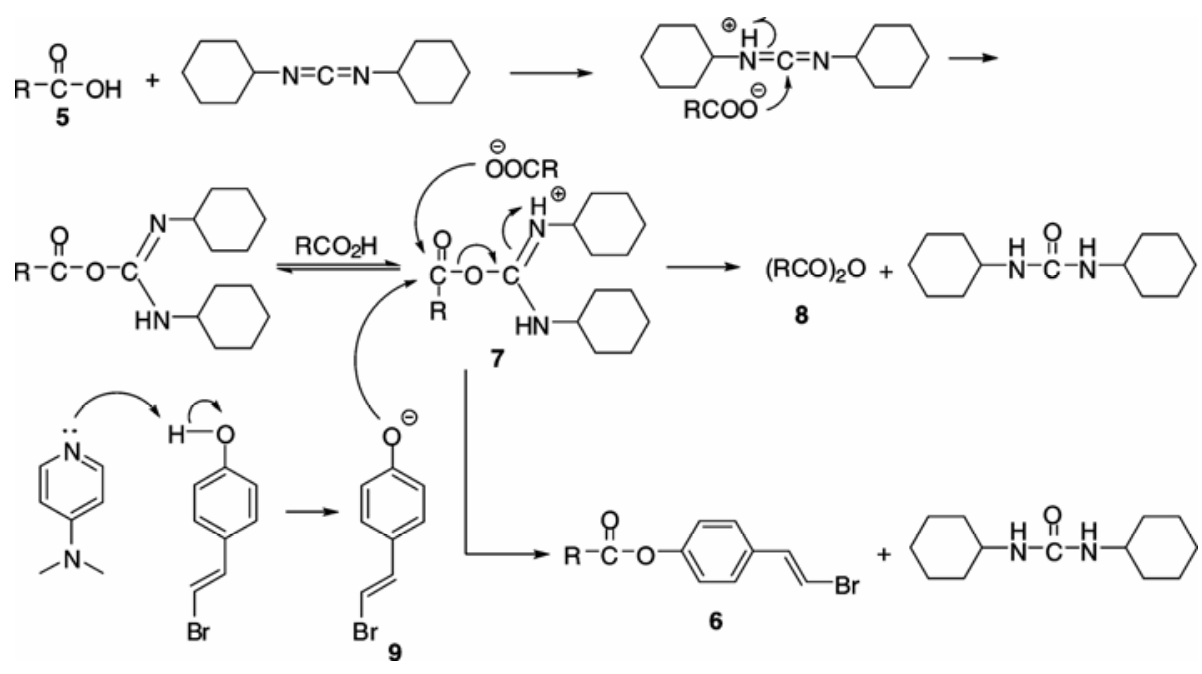

Scheme 3. Mechanistic hypothesis for the formation of functionalized $(E)-2-$ arylvinyl bromides 6 .

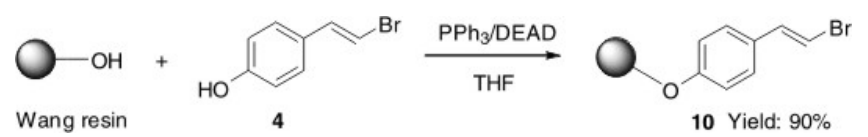

Scheme 4. (E)-4-(2-bromovinyl)phenol 3 linked to Wang resin.

carbonyl- and 4-aryloxycarbonyl-(E)- $\beta$-arylvinyl bromides. A wide range of functional groups such as $-\mathrm{CO}_{2} \mathrm{R}(5 \mathbf{e}),-\mathrm{CHO}(\mathbf{5 f})$, conjugate diene (5b), ether (5c), styryl (5d), vinyl bromide (5g-5h) were found tolerant to this condition. Even the molecule contained heterocyclic rings such as pyridyl $(\mathbf{5 i}-\mathbf{5 j})$, furyl (5k), thienyl (5l), the reaction stereoselectively proceeded in high yields. The structures of these compounds has been elucidated by elemental analysis and spectral (IR, ${ }^{1} \mathrm{H}$ NMR, ${ }^{13} \mathrm{C}$ NMR, MS) data, as shown in appendix $\mathrm{A}$.

A mechanistic hypothesis for the esterification of (E)-4-(2-bromovinyl)phenol (4) with various acids is proposed in scheme 3. (E)-4-(2-bromovinyl)phenol is firstly converted to the corresponding anion 8 in the presence of DMAP. And then anion 8 attacks the carbonyl carbon atom of intermediate 7 generated from DCC with acid $\mathbf{5}$ to form the target molecule $\mathbf{6}$. The key point of this mechanism is the formation of (E)-4-(2-bromovinyl)phenol anion 8 should be ahead of the intermediate 7. Otherwise the acid anion will attack the carbonyl carbon atom of intermediate 7 and generate by-product $\mathbf{8}$, leading low yields (scheme 3).

Further, (E)-4-(2-bromovinyl)phenol (4) could be attached to the benzyl alcohol of Wang resin using a
$\mathrm{PPh}_{3} /$ DEAD coupling procedure at ambient temperature to afford functionalized $(E)$-2-arylvinyl bromide ether 7 in high yield (scheme 4 ).

The Wang resin-bound ester $\mathbf{1 0}$ carrying an active bromovinyl group can be applied to combinatorial and solid phase organic synthesis, which are efficient techniques for the production of combinatorial libraries and are extensively used by the pharmaceutical and the agricultural industries. Further, 7 is an excellent substrate for Stille, Suzuki and Heck reaction. ${ }^{1}$

\section{Conclusions}

In summary, we have developed a novel and convenient synthetic route for preparing high functionalized (E)-2-arylvinyl bromides bearing a wide range of functional groups. These functionalized (E)-2arylvinyl bromides are important synthetic targets and widely used in the preparation of pharmaceuticals, functional polymeric material and natural products. $^{15}$

\section{Appendix A}

(E)-4-(2-Bromovinyl)phenyl palmitate 6a: White solid, m.p. $72 \cdot 5-73 \cdot 0^{\circ} \mathrm{C}$. IR (KBr): 1739,1283 , 1087, 935, $777 \mathrm{~cm}^{-1} .{ }^{1} \mathrm{H}$ NMR $\left(300 \mathrm{MHz}, \mathrm{CDCl}_{3}\right.$, $\delta \mathrm{ppm}): 0.88(3 \mathrm{H}, t, J=6.3 \mathrm{~Hz}), 1.20-1.40(24 \mathrm{H}$, $m), 1.70-1.77(2 \mathrm{H}, m), 2.55(2 \mathrm{H}, t, J=7.2 \mathrm{~Hz}), 6.73$ $(1 \mathrm{H}, d, J=14 \cdot 1 \mathrm{~Hz}), 7 \cdot 03-7 \cdot 1(3 \mathrm{H}, m), 7 \cdot 31(2 \mathrm{H}, d$, $J=8.7 \mathrm{~Hz}) .{ }^{13} \mathrm{C}$ NMR $\left(75 \mathrm{MHz}, \mathrm{CDCl}_{3}, \delta \mathrm{ppm}\right)$ : $14 \cdot 1,22 \cdot 7,24 \cdot 9,29 \cdot 1,29 \cdot 2,29 \cdot 4,29 \cdot 43,29 \cdot 6,29 \cdot 7$, 
$31 \cdot 9,34 \cdot 4,106 \cdot 5,122 \cdot 0,127 \cdot 1,133 \cdot 5,136 \cdot 2,150 \cdot 6$, 172.1. EI-MS: $m / z(\%): 438\left((\mathrm{M}+2)^{+}, 18\right), 436$ $\left(\mathrm{M}^{+}, 19\right), 200$ (100), 198 (99). Anal. calcd. for $\mathrm{C}_{24} \mathrm{H}_{37} \mathrm{BrO}_{2}$ : C, 65.89; $\mathrm{H}, 8.53 ; \mathrm{Br}, 18 \cdot 27$. found: $\mathrm{C}$, $65 \cdot 82 ; \mathrm{H}, 8 \cdot 58 ; \mathrm{Br}, 18 \cdot 32$.

$(2 E, 4 E)-4-((E)-2-B r o m o v i n y l) p h e n y l$ hexa-2,4dienoate 6b: White solid, m.p. $120 \cdot 4-121.4^{\circ} \mathrm{C}$. IR (KBr): 1724, 1215, 1077, 935, $777 \mathrm{~cm}^{-1} .{ }^{1} \mathrm{H}$ NMR $\left(300 \mathrm{MHz}, \mathrm{CDCl}_{3}, \delta \mathrm{ppm}\right): 1.90(3 \mathrm{H}, d, J=4.5 \mathrm{~Hz})$, $5.95(1 \mathrm{H}, d, J=15.6 \mathrm{~Hz}), 6 \cdot 20-6.34(2 \mathrm{H}, m), 6.73$ $(1 \mathrm{H}, d, J=14.1 \mathrm{~Hz}), 7.09 \quad(3 \mathrm{H}, d, J=8.4 \mathrm{~Hz})$, $7.31(2 \mathrm{H}, d, J=8.4 \mathrm{~Hz}), 7.39-7.48(1 \mathrm{H}, m) .{ }^{13} \mathrm{C}$ NMR (75 MHz, $\left.\mathrm{CDCl}_{3}, \delta \mathrm{ppm}\right): 18 \cdot 7,106 \cdot 5,117 \cdot 7$, $122 \cdot 0,127 \cdot 0,129 \cdot 7,133 \cdot 4,136 \cdot 3,140 \cdot 9,147 \cdot 2$, 150.7, 165.4. EI-MS: $m / z(\%): 294\left((\mathrm{M}+2)^{+}, 15\right)$, $292\left(\mathbf{M}^{+}, 15\right), 200$ (100), 198 (99). Anal. calcd. for $\mathrm{C}_{14} \mathrm{H}_{13} \mathrm{BrO}_{2}$ : C, 57.36; $\mathrm{H}, 4.47 ; \mathrm{Br}, 27 \cdot 26$. found: $\mathrm{C}$, $57 \cdot 32 ; \mathrm{H}, 4 \cdot 50 ; \mathrm{Br}, 27 \cdot 28$.

(E)-4-(2-Bromovinyl)phenyl 2-phenoxyacetate $\mathbf{6 c}$ : White solid, m.p. $204 \cdot 5-205 \cdot 5^{\circ} \mathrm{C}$. IR (KBr): 1729 , 1240, 1092, 935, $746 \mathrm{~cm}^{-1}$. ${ }^{1} \mathrm{H}$ NMR $(300 \mathrm{MHz}$, $\left.\mathrm{CDCl}_{3}, \delta \mathrm{ppm}\right): 4.89(2 \mathrm{H}, \quad s), 6.75 \quad(1 \mathrm{H}, d$, $J=14 \cdot 1 \mathrm{~Hz}), 6 \cdot 91-7 \cdot 15(6 \mathrm{H}, m), 7 \cdot 23-7 \cdot 36(4 \mathrm{H}, m)$.

${ }^{13} \mathrm{C}$ NMR $\left(75 \mathrm{MHz}, \mathrm{CDCl}_{3}, \delta \mathrm{ppm}\right): 64 \cdot 5,108 \cdot 2$, $114.4,120.9,121.4,122.0,127.5,129.4,129.5$, 133.64, 135.8, 157.4, 157.7, 167.6. EI-MS: $m / z(\%)$ : $334\left((\mathrm{M}+2)^{+}, 19\right), 332\left(\mathrm{M}^{+}, 20\right), 200(100), 198$ (99). Anal. calcd. for $\mathrm{C}_{16} \mathrm{H}_{13} \mathrm{BrO}_{3}: \mathrm{C}, 57.68 ; \mathrm{H}, 3.93$; $\mathrm{Br}, 23.98$. found: C, 57.74; H, 3.96; Br, 23.96.

4-((E)-2-Bromovinyl)phenyl cinnamate 6d: White solid, m.p. $151 \cdot 5-152 \cdot 0^{\circ} \mathrm{C}$. IR (KBr): 1714, 1276, $1103,935,746 \mathrm{~cm}^{-1} .{ }^{1} \mathrm{H}$ NMR $\left(300 \mathrm{MHz}, \mathrm{CDCl}_{3}\right.$, $\delta \mathrm{ppm}): 6.63(1 \mathrm{H}, d, J=8.1 \mathrm{~Hz}), 6.76(1 \mathrm{H}, d$, $J=8.4 \mathrm{~Hz}), \quad 7.09-7.16 \quad(3 \mathrm{H}, \quad m), \quad 7.35 \quad(2 \mathrm{H}, d$, $J=8.4 \mathrm{~Hz}), 7.42-7.45(3 \mathrm{H}, m), 7.58-7.60(2 \mathrm{H}, m)$, $7.88(1 \mathrm{H}, d, J=13.8 \mathrm{~Hz}) .{ }^{13} \mathrm{C}$ NMR $(75 \mathrm{MHz}$, $\left.\mathrm{CDCl}_{3}, \delta \mathrm{ppm}\right): 106 \cdot 6,117 \cdot 0,122 \cdot 0,127 \cdot 1,128 \cdot 3$, $129 \cdot 0,130 \cdot 8,133 \cdot 6,134 \cdot 1,136 \cdot 2,134 \cdot 1,136 \cdot 2$, 146.8, 150.6, 165.2. EI-MS: $m / z(\%): 330\left((\mathrm{M}+2)^{+}\right.$, 20), $328\left(\mathrm{M}^{+}, 21\right), 200$ (100), 198 (99). Anal. calcd. for $\mathrm{C}_{17} \mathrm{H}_{13} \mathrm{BrO}_{2}$ : C, 62.03; H, 3.98; $\mathrm{Br}, 24.27$. found: C, $62 \cdot 07 ; \mathrm{H}, 3 \cdot 41 ; \mathrm{Br}, 24 \cdot 30$.

Methyl-4-((E)-3-(4-((E)-2-bromovinyl)phenoxy)-3oxoprop-1-enyl)benzoate 6e: White solid, m.p. $164.2-164.9^{\circ} \mathrm{C}$. IR (KBr): 1729, 1291, 1153, 940, $767 \mathrm{~cm}^{-1} .{ }^{1} \mathrm{H}$ NMR $\left(300 \mathrm{MHz}, \mathrm{CDCl}_{3}, \delta \mathrm{ppm}\right): 3.95$ $(3 \mathrm{H}, s), 6.68-6.78(2 \mathrm{H}, m), 7.09-7.37(5 \mathrm{H}, m)$,
7.65-7.67 $(2 \mathrm{H}, m), 7.89(1 \mathrm{H}, d, J=15.0 \mathrm{~Hz}), 8 \cdot 10$ $(2 \mathrm{H}, d, J=8 \cdot 1 \mathrm{~Hz}) .{ }^{13} \mathrm{C}$ NMR $\left(75 \mathrm{MHz}, \mathrm{CDCl}_{3}\right.$, $\delta \mathrm{ppm}): 52 \cdot 3,106 \cdot 1,119.5,121.9,127 \cdot 1,128 \cdot 1$, $130 \cdot 2,131 \cdot 8,133 \cdot 8,136 \cdot 2,138 \cdot 2,145 \cdot 3,150 \cdot 5$, 164.3, 165.9. EI-MS: $m / z(\%): 388\left((\mathrm{M}+2)^{+}, 20\right)$, $386\left(\mathrm{M}^{+}, 20\right), 200$ (100), 198 (99). Anal. calcd. for $\mathrm{C}_{19} \mathrm{H}_{15} \mathrm{BrO}_{4}$ : C, 58.93; H, 3.90; $\mathrm{Br}, 20.64$. found: $\mathrm{C}$, $58 \cdot 90 ; \mathrm{H}, 3 \cdot 88 ; \mathrm{Br}, 20 \cdot 68$.

(E)-4-(2-Bromovinyl)phenyl-4-formylbenzoate $\mathbf{6 f}$ : White solid, m.p. $129 \cdot 0-130 \cdot 0^{\circ} \mathrm{C}$. IR ( $\left.\mathrm{KBr}\right)$ : 1734, 1266, 1067, 935, $756 \mathrm{~cm}^{-1} .{ }^{1} \mathrm{H}$ NMR $(300 \mathrm{MHz}$, $\left.\mathrm{CDCl}_{3}, \delta \mathrm{ppm}\right): 6.79(1 \mathrm{H}, d, J=13.7 \mathrm{~Hz}), 7 \cdot 13(1 \mathrm{H}$, $d, J=13.7 \mathrm{~Hz}), 7.21(2 \mathrm{H}, d, J=8.7 \mathrm{~Hz}), 7.39(2 \mathrm{H}$, $d, J=8.7 \mathrm{~Hz}), 8.04(2 \mathrm{H}, d, J=8.3 \mathrm{~Hz}), 8.37(2 \mathrm{H}, d$, $J=8.3 \mathrm{~Hz}), 10 \cdot 16(1 \mathrm{H}, s) .{ }^{13} \mathrm{C}$ NMR $(75 \mathrm{MHz}$, $\left.\mathrm{CDCl}_{3}, \delta \mathrm{ppm}\right): 107 \cdot 0,121 \cdot 3,121 \cdot 9,127 \cdot 2,129 \cdot 6$, $130 \cdot 8,134 \cdot 1,136 \cdot 1,139 \cdot 7,150 \cdot 5,164 \cdot 5,191 \cdot 4$. EIMS: $m / z(\%): 332\left((\mathrm{M}+2)^{+}, 17\right), 330\left(\mathbf{M}^{+}, 18\right), 200$ (100), 198 (99). Anal. calcd. for $\mathrm{C}_{16} \mathrm{H}_{11} \mathrm{BrO}_{3}$ : C, $58.03 ; \mathrm{H}, 3.35 ; \mathrm{Br}, 24 \cdot 13$. found: $\mathrm{C}, 58.06 ; \mathrm{H}, 3.32$; $\mathrm{Br}, 24 \cdot 18$.

4-((E)-2-Bromovinyl)phenyl 4-((Z)-2-bromovinyl) benzoate 6g: Light yellow solid, m.p. 118.5$119 \cdot 0^{\circ} \mathrm{C}$. IR (KBr) $1724,1261,1072,935,772 \mathrm{~cm}^{-1}$. ${ }^{1} \mathrm{H}$ NMR $\left(300 \mathrm{MHz}, \mathrm{CDCl}_{3}, \delta \mathrm{ppm}\right): 6.62(1 \mathrm{H}, d$, $J=8 \cdot 1 \mathrm{~Hz}), 6.77(1 \mathrm{H}, d, J=14 \cdot 1 \mathrm{~Hz}), 7 \cdot 10-7 \cdot 21$ $(4 \mathrm{H}, m), 7.37(2 \mathrm{H}, d, J=8.4 \mathrm{~Hz}), 7.81(2 \mathrm{H}, d$, $J=8.4 \mathrm{~Hz}), 8.20(2 \mathrm{H}, d, J=8.4 \mathrm{~Hz}) .{ }^{13} \mathrm{C}$ NMR $\left(75 \mathrm{MHz}, \mathrm{CDCl}_{3}, \delta \mathrm{ppm}\right): 106 \cdot 7,109 \cdot 3,122 \cdot 1$, $126 \cdot 1,127 \cdot 2,129 \cdot 1,130 \cdot 1,131 \cdot 5,133 \cdot 8,136 \cdot 2$, 140.1, 150.7, 164.6. EI-MS: $m / z(\%): 408\left((\mathrm{M}+2)^{+}\right.$, 16), $406\left(\mathrm{M}^{+}, 16\right), 200$ (100), 198 (99). Anal. calcd. for $\mathrm{C}_{17} \mathrm{H}_{12} \mathrm{Br}_{2} \mathrm{O}_{2}$ : C, 50.03; $\mathrm{H}, 2.96 ; \mathrm{Br}, 39.16$. found: C, 50.04; $\mathrm{H}, 3 \cdot 02 ; \mathrm{Br}, 39 \cdot 21$.

4-((E)-2-Bromovinyl)phenyl 4-((E)-2-bromovinyl) benzoate $6 \mathbf{h}$ : White solid, m.p. $136 \cdot 5-137 \cdot 5^{\circ} \mathrm{C}$. IR (KBr) $1724,1271,1072,935,746 \mathrm{~cm}^{-1} .{ }^{1} \mathrm{H}$ NMR $\left(300 \mathrm{MHz}, \mathrm{CDCl}_{3}, \delta \mathrm{ppm}\right): 6.77(1 \mathrm{H}, d, J=$ $14.0 \mathrm{~Hz}), 6.99(1 \mathrm{H}, d, J=14.0 \mathrm{~Hz}), 7 \cdot 10-7.20(4 \mathrm{H}$, $m), 7 \cdot 35-7.45(4 \mathrm{H}, m), 8 \cdot 15(2 \mathrm{H}, d, J=8 \cdot 7 \mathrm{~Hz}) \cdot{ }^{13} \mathrm{C}$ NMR (75 MHz, $\left.\mathrm{CDCl}_{3}, \delta \mathrm{ppm}\right): 106 \cdot 8,110 \cdot 1,122 \cdot 1$, $126 \cdot 2,127 \cdot 2,128.7,130.7,133.8,136.2,138 \cdot 2$, $140 \cdot 8,150 \cdot 7,164 \cdot 5$. EI-MS: $m / z(\%): 408\left((\mathrm{M}+2)^{+}\right.$, 16), $406\left(\mathrm{M}^{+}, 17\right), 200$ (100), 198 (99). Anal. calcd. for $\mathrm{C}_{17} \mathrm{H}_{12} \mathrm{Br}_{2} \mathrm{O}_{2}: \mathrm{C}, 50.03 ; \mathrm{H}, 2.96 ; \mathrm{Br}, 39 \cdot 16$. found: C, 50.05; H, 3.01; $\mathrm{Br}, 39 \cdot 20$.

(E)-4-(2-Bromovinyl)phenyl nicotinate $\mathbf{6 i}$ : Light yellow solid, m.p. $126 \cdot 0-127 \cdot 0^{\circ} \mathrm{C}$. IR (KBr): 1734 , 
1276, 1076, 935, $777 \mathrm{~cm}^{-1} .{ }^{1} \mathrm{H}$ NMR $(300 \mathrm{MHz}$, $\left.\mathrm{CDCl}_{3}, \delta \mathrm{ppm}\right): 6.79(1 \mathrm{H}, d, J=14.3 \mathrm{~Hz}), 7 \cdot 13(1 \mathrm{H}$, $d, J=14.3 \mathrm{~Hz}), 7.21(2 \mathrm{H}, d, J=8.1 \mathrm{~Hz}), 7.38(2 \mathrm{H}$, $d, J=8.1 \mathrm{~Hz}), 7.46-7.50(1 \mathrm{H}, t), 8.45(1 \mathrm{H}, d$, $J=8 \cdot 1 \mathrm{~Hz}), 8.87(1 \mathrm{H}, d, J=5 \cdot 1 \mathrm{~Hz}), 9.40(1 \mathrm{H}, s)$. ${ }^{13} \mathrm{C}$ NMR $\left(75 \mathrm{MHz}, \mathrm{CDCl}_{3}, \delta \mathrm{ppm}\right): 107 \cdot 0,122 \cdot 0$, $123 \cdot 5,125 \cdot 4,127 \cdot 2,134 \cdot 1,136 \cdot 1,137 \cdot 6,150 \cdot 3$, 151.3, 154.0, 163.7. EI-MS: $m / z(\%): 305\left((\mathrm{M}+2)^{+}\right.$, 21), $303\left(\mathrm{M}^{+}, 22\right), 200$ (100), 198 (99). Anal. calcd. for $\mathrm{C}_{14} \mathrm{H}_{10} \mathrm{BrNO}_{2}$ : C, 55.29; $\mathrm{H}, 3.31 ; \mathrm{Br}, 26 \cdot 27$. found: $\mathrm{C}, 55 \cdot 34 ; \mathrm{H}, 3 \cdot 33 ; \mathrm{Br}, 26 \cdot 21$.

(E)-4-(2-Bromovinyl)phenyl isonicotinate $\mathbf{6 j}$ : Light yellow solid, m.p. $124 \cdot 0-124 \cdot 6^{\circ} \mathrm{C}$. IR ( $\left.\mathrm{KBr}\right)$ : 1734 , 1271, 1087, 935, $746 \mathrm{~cm}^{-1}$. ${ }^{1} \mathrm{H}$ NMR $(300 \mathrm{MHz}$, $\left.\mathrm{CDCl}_{3}, \delta \mathrm{ppm}\right): 6.79(1 \mathrm{H}, d, J=14.3 \mathrm{~Hz}), 7 \cdot 13(1 \mathrm{H}$, $d, J=14.3 \mathrm{~Hz}), 7 \cdot 20(2 \mathrm{H}, d, J=8.3 \mathrm{~Hz}), 7 \cdot 38(2 \mathrm{H}$, $d, J=8.3 \mathrm{~Hz}), 7.50(2 \mathrm{H}, d, J=5.7 \mathrm{~Hz}), 8.87(2 \mathrm{H}, d$, $J=5.7 \mathrm{~Hz}) .{ }^{13} \mathrm{C}$ NMR $\left(75 \mathrm{MHz}, \mathrm{CDCl}_{3}, \delta \mathrm{ppm}\right)$ : $107 \cdot 1,121 \cdot 8,123 \cdot 2,127 \cdot 3,134 \cdot 2,136 \cdot 0,136 \cdot 6$, 150.2, 150.8, 163.6. EI-MS: $m / z(\%): 305\left((\mathrm{M}+2)^{+}\right.$, 20), $303\left(\mathrm{M}^{+}, 21\right), 200$ (100), 198 (99). Anal. calcd. for $\mathrm{C}_{14} \mathrm{H}_{10} \mathrm{BrNO}_{2}$ : C, 55.29; $\mathrm{H}, 3.31 ; \mathrm{Br}, 26.27$. found: C, 55.35; H, 3.34; $\mathrm{Br}, 26 \cdot 20$.

(E)-4-(2-Bromovinyl) phenyl furan-2-carboxylate $\mathbf{6 k}$ : White solid, m.p. $100 \cdot 5-101 \cdot 0^{\circ} \mathrm{C}$. IR (KBr): 1734 , 1275, 1092, 930, $762 \mathrm{~cm}^{-1}$. ${ }^{1} \mathrm{H}$ NMR $(300 \mathrm{MHz}$, $\left.\mathrm{CDCl}_{3}, \delta \mathrm{ppm}\right): 6.60-6.61(1 \mathrm{H}, m), 6.76(1 \mathrm{H}, d$, $J=14 \cdot 1 \mathrm{~Hz}), 7 \cdot 11(1 \mathrm{H}, d, J=14 \cdot 1 \mathrm{~Hz}), 7 \cdot 19(2 \mathrm{H}, d$, $J=8.4 \mathrm{~Hz}), 7.34-7.40(3 \mathrm{H}, q), 7.69(1 \mathrm{H}, s) \cdot{ }^{13} \mathrm{C}$ NMR (75 MHz, $\left.\mathrm{CDCl}_{3}, \delta \mathrm{ppm}\right): 106 \cdot 8,112 \cdot 2,119 \cdot 6$, $121.9,127 \cdot 2,133 \cdot 9,136 \cdot 1,143 \cdot 8,147 \cdot 3,150 \cdot 0$, 156.6. EI-MS: $m / z(\%): 294\left((\mathrm{M}+2)^{+}, 15\right), 292$ $\left(\mathrm{M}^{+}, 15\right), 200$ (100), 198 (99). Anal. calcd. for $\mathrm{C}_{13} \mathrm{H}_{9} \mathrm{BrO}_{3}$ : C, 53.27; H, 3.09; $\mathrm{Br}, 27 \cdot 26$. found: $\mathrm{C}$, $53 \cdot 33 ; \mathrm{H}, 3 \cdot 02 ; \mathrm{Br}, 27 \cdot 23$.

(E)-4-(2-Bromovinyl)phenyl thiophene-2-carboxylate 61: Yellow solid, m.p. $114 \cdot 9-115 \cdot 5^{\circ} \mathrm{C}$. IR (KBr): 1719, 1272, 1080, 935, $767 \mathrm{~cm}^{-1} .{ }^{1} \mathrm{H}$ NMR $\left(300 \mathrm{MHz}, \quad \mathrm{CDCl}_{3}, \quad \delta \mathrm{ppm}\right): 6.77 \quad(1 \mathrm{H}, \quad \mathrm{d}$, $J=14.3 \mathrm{~Hz}), 7 \cdot 12(1 \mathrm{H}, d, J=14.3 \mathrm{~Hz}), 7 \cdot 17-7 \cdot 21$ $(3 \mathrm{H}, m), 7.36(2 \mathrm{H}, d, J=8.1 \mathrm{~Hz}), 7.67(1 \mathrm{H}, m)$, 7.98-7.99 $(1 \mathrm{H}, m) .{ }^{13} \mathrm{C}$ NMR $\left(75 \mathrm{MHz}, \mathrm{CDCl}_{3}\right.$, $\delta \mathrm{ppm}): 106 \cdot 8,121 \cdot 7,127 \cdot 1,128 \cdot 1,129 \cdot 6,132 \cdot 0$,
$133 \cdot 7,134 \cdot 8,136 \cdot 2,150 \cdot 4,160 \cdot 4$. EI-MS: $m / z(\%)$ : $310\left((\mathrm{M}+2)^{+}, 14\right), 308\left(\mathrm{M}^{+}, 15\right), 200(100), 198$ (99). Anal. calcd. for $\mathrm{C}_{13} \mathrm{H}_{9} \mathrm{BrO}_{2} \mathrm{~S}$ : C, 50.50; H, $2.93 ; \mathrm{Br}, 25.84$. found: $\mathrm{C}, 50.45 ; \mathrm{H}, 2.95 ; \mathrm{Br}, 25.85$.

\section{Acknowledgements}

The work was supported by the Natural Science Foundation of Shanghai, China (No. 06ZR14085) and by the Scientific Research Foundation for the Returned Overseas Chinese Scholars, State Education Ministry. We would like to thank the Instrumental Analysis Center, Tongji University, China, for Instrumental Analysis.

\section{References}

1. (a) Meijere A D, Diederich F and Stille J K 2004 Metal-catalysed cross-coupling Reactions (New York: Wiley-VCH) pp 1-40; (b) Negishi E I 2002 Handbook of organo palladium chemistry for organic synthesis (New York: Wiley Interscience) pp 335-408

2. (a) Alzeer J, Chollet J and Heinze-Krauss 2000 Ind. J. Med. Chem. 43 560; (b) Kitamura T, Aoyagi Y and Fujiwara Y 1999 Chem. Lett. 281271

3. Graven A, Jorgensen K A and Dahl S J 1994 Org. Chem. 593543

4. Shantanu C and Sujit R 1997 J. Org. Chem. 62199

5. Dinabandhu N and Sujit R 2000 Tetrahedron 561369

6. (a) Kuang C X, Senboku H and Tokuda M 2000 Synlett. 1439; (b) Kuang C X, Senboku H and Tokuda M 2005 Synthesis 1319

7. (a) Homsi F and Rousseau G $1999 \mathrm{~J}$. Org. Chem. 64 81; (b) Homsi F and Rousseau G 1999 Tetrahedron Lett. 401495

8. Yu L, Chen B and Huang X 2007 Chin. Chem. Lett. 18121

9. Roy S C, Guin C and Maiti G 2001 Tetrahedron Lett. 429253

10. Telvekar V N, Arote N D and Herlekar O P 2005 Synlett. 2495

11. Sinha J, Layek S and Bhattacharjee M 2001 Chem. Commun. 1916

12. Ye C F and Shreeve J M 2004 J. Org. Chem. 69 8561

13. Choudary B M, Someshwar T and Kantam M L 2004 Catal. Commun. 5215

14. Boiadjiev S E and Lightner D A 1996 Tetrahedron: Asymmetry 72825

15. Katritzky A R 2004 Chem. Rev. 1042125 